\title{
XIII International Symposium on Luminescence Spectrometry (XIII ISLS)
}

\author{
Aldo Roda \\ Published online: 29 April 2009 \\ (C) Springer-Verlag 2009
}

Luminescence detection techniques have proved to be among the most powerful, because of their rapidity, sensitivity, and suitability for miniaturized and biosensor formats. Most analytical and bioanalytical methods nowadays employ spectroscopic emission signal detection, for example fluorescence, time-resolved fluorescence, fluorescence polarization, fluorescence resonance energy transfer, electrochemiluminescence, chemiluminescence, bioluminescence, and bioluminescence resonance energy transfer, spanning across a wide range of applications. This trend has been fostered by the development of several new luminescent probes, assay designs and instrumental technologies that brought new detection capabilities.

The International Symposium on Luminescence Spectrometry (ISLS) started in 1984 in Ghent (Belgium), and was organized by Willy R.G. Baeyens as a biennial international forum for scientists involved in all aspects of luminescence spectrometry, to highlight recent trends and advances in this field.

The XIII ISLS, organized by Aldo Roda (Professor of Analytical Chemistry at the Faculty of Pharmacy, University of Bologna - Alma Mater Studiorum), was held on September 7-11, 2008, in Bologna (Italy), at the Complex of San Giovanni in Monte of the University of Bologna. The Symposium was attended by more than 250 scientists representing 30 countries from all over the world and was supported by several exhibitors and sponsors. The scientific program (40 invited lectures, 55 oral communications, and

\section{A. Roda $(\triangle)$}

Analytical-Bioanalytical Laboratory,

Department of Pharmaceutical Sciences,

Alma Mater Studiorum-University of Bologna,

Via Belmeloro 6,

40126 Bologna, Italy

e-mail: aldo.roda@unibo.it
161 posters) was a blend of basic research and applications of luminescence in a wide range of fields, including the biosciences, cultural heritage preservation, and pharmaceutical, environmental, food, and forensic analysis. Three workshops were dedicated to the discussion of specific applications of luminescence techniques in fields of particular interest:

- "Luminescence-based detection methods to improve food safety" was focused on the presentation of novel rapid, portable, automatic, and low-cost technologies for food-safety monitoring, exploiting luminescence detection.

- "Luminescence techniques in science for conservation of cultural heritage" was dedicated to the luminescent techniques used to characterize the complex material systems of works of art and to investigate their degradation processes.

- "Light on the crime scene: forensic applications" gathered contributions from academics involved in the development of new analytical tools to "illuminate" the crime scene, and representatives of police forensic science service departments from different countries, who employ such tools in crime-scene analysis. New analytical tools and their practical use in everyday cases were presented.

This special issue of Analytical and Bioanalytical Chemistry contains nineteen papers that reflect the main topics covered at the XIII ISLS.

A large number of oral and poster presentations were devoted to the coupling of luminescence detection with separation techniques. This special issue of Analytical and Bioanalytical Chemistry includes papers spanning from method development and optimization (e.g., novel sample-preparation techniques) or focused applications, to 
original approaches combining luminescence detection with separation techniques employing antibodies and molecular imprinted polymers as recognition systems.

Bioanalytical luminescence spectrometry applications, which usually represent a significant fraction of the contributions in ISLS symposia, are illustrated by a number of papers ranging from the use of chemiluminescence imaging techniques for localization of biomarkers in tissue samples to use of fluorescence techniques for measurement of enzyme activities or interactions of biological macromolecules.

The presence of a workshop dedicated to the application of luminescence detection in the science of characterization and conservation of cultural heritage promoted the participation of a much greater number of scientists involved in this field, compared with previous ISLS meetings. The development of non-invasive or minimally invasive techniques taking advantage of the high selectivity and sensitivity of luminescence detection were important advances in this field.

Environmental analysis is the focus of other papers on the development of luminescence-based analytical methods to enable highly sensitive detection of pollutants in raw samples, without the need of pre-analytical concentration or purification methods.

The XIV ISLS will be held in Prague, Czech Republic, between 13 and 16 July 2010 and organized by P. Solich. More detailed information concerning the XIV ISLS is available at www.faf.cuni.cz/isls2010.

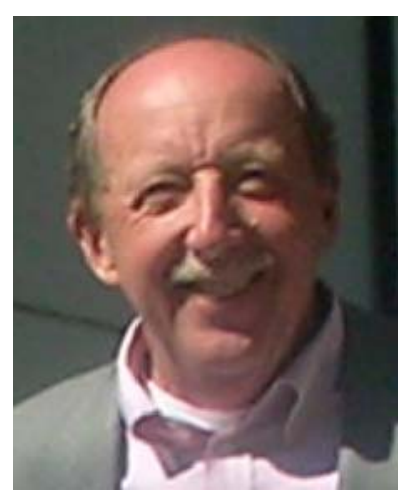

Aldo Roda is Professor of Analytical Chemistry at Bologna University. His main research interests include the development of ultrasensitive bioanalytical methods employing bioluminescence, chemiluminescence, and electrochemiluminescence detection, for example immunoassays, gene hybridization assays, imaging techniques for localization of proteins and nucleic acids in cells and tissues, genetically engineered whole-cell biosensors, and whole-animal imaging. Proteomic studies for biomarker discovery based on liquid chromatography and mass spectrometry are also performed in his laboratory 